Informes de la Construcción

Vol. 67, EXTRA-1, ntoo4

marzo 2015

ISSN-L: 0020-0883

doi: http://dx.doi.org/10.3989/ic.14.066

NOTA TÉCNICA

\title{
Proyecto SYMBCITY del Solar Decathlon Europe 2014: redensificación sostenible como forma de intervención urbana en tiempos de crisis
}

\author{
SYMBCITY Project in Solar Decathlon Europe 2014: sustainable \\ redensification as a way of urban intervention in times of crisisA
}

$\underline{\text { A. Baño Nieva }^{(*)}}$, F. Castilla Pascual ${ }^{(* *)}$, F. Estirado Oliet ${ }^{(*)}$

RESUMEN

Partiendo del concepto de «redensificación sostenible» se propone la colonización o reordenación de espacios disponibles sobre la cubierta, bajos o entre medianeras de edificaciones existentes, para usos demandados en cada barrio, y el desarrollo de sus fachadas, generando un nuevo conjunto urbano que en su asociación origina un organismo simbiótico beneficiando a todas las partes implicadas. Este aumento de edificabilidad y su explotación permitirá la financiación de las mejoras energéticas y de accesibilidad de la edificación ocupada que cede su terreno. Este proyecto forma parte del desarrollo del prototipo del equipo Plateau Team, formado por las Universidades de Alcalá y Castilla-La Mancha, seleccionado para la competición «Solar Decathlon 2014». Esta apuesta innovadora de regeneración urbana incluye el análisis urbano, social, técnico y económico, busca nuevos lugares de oportunidad y crea una simbiosis entre lo nuevo y lo antiguo mejorando la eficiencia energética del parque de viviendas existente.

Palabras clave: Redensificación sostenible; simbiosis; regeneración urbana; eficiencia energética; rehabilitación.

\begin{abstract}
Based on the concept of «sustainable redensification» we propose the colonization or refurbishment of available spaces on the roof, lower floors or between walls of existing buildings, for demanded uses in each city district, and the retrofit of its facades, generating a new urban unit that gives birth to a symbiotic organism benefitting all parties involved. This increase of density and its exploitation will allow financing the energy and accessibility improvement of the occupied building that shares its land. This project is part of the development of the prototype by the Plateau Team, formed by Alcala University and Castilla-La Mancha University, selected team for the «Solar Decathlon 2014» competition. This innovative urban regeneration proposal includes urban, social, technical and economic analysis, searches for new places of business opportunities, and creates a symbiosis between the new and the old to improve the energy efficiency of existing buildings.
\end{abstract}

Keywords: Sustainable redensification; symbiosis; urban regeneration; energy efficiency; retrofit.

(*) Departamento de Arquitectura, Universidad de Alcalá. Madrid (España).

(**) Departamento de Ingeniería Civil y de la Edificación, Universidad de Castilla-La Mancha. Ciudad Real (España). Persona de contacto/Corresponding author: antonio.banno@uah.es (A. Baño Nieva)

Cómo citar este artículo/Citation: Baño Nieva, A., Castilla Pascual, F., Estirado Oliet, F. (2015). Proyecto SYMBICTY del Solar Decathlon Europe 2014: redensificación sostenible como forma de intervención urbana en tiempos de crisis. Informes de la Construcción, 67(EXTRA-1): ntoo4, doi: http://dx.doi.org/10.3989/ic.14.066.

Licencia/License: Salvo indicación contraria, todos los contenidos de la edición electrónica de Informes de la Construcción se distribuyen bajo una licencia de uso y distribución Creative Commons Reconocimiento no Comercial 3.o. España (cc-by-nc). 


\section{PRESENTACIÓN}

El proyecto SYMBCITY nace como soporte conceptual de la candidatura presentada por el equipo Plateau Team (de las Universidades de Alcalá de Henares y de Castilla la Mancha) para su participación en el Concurso Internacional Solar Decathlon Europe 2014 (SDE2014). La decisión de la Organización de centrar las propuestas de este año en el ámbito urbano permite explorar sendas no recorridas en ediciones anteriores.

Desde la trasposición a la ciudad del concepto «SIMBIOSIS», como resultado de la colaboración entre organismos que obtienen un beneficio común, se muestra la necesidad que tiene la ciudad actual de recurrir a soluciones imaginativas que permitan reponer el valor perdido de nuestra edificación, en base a las actuales exigencias de confort, habitabilidad y sostenibilidad. El organismo «huésped» proporcionaría tecnología actual y con ello la posibilidad de adaptación a los nuevos tiempos. El «anfitrión», aporta un suelo urbano amortizado y un entorno urbano consolidado.

El proyecto completo se desarrolla en dos ámbitos complementarios, simbióticos. Por un lado, el motor y escaparate del proyecto es el Concurso Internacional SDE2014 y la construcción del prototipo de vivienda eficiente que debe someterse a las pruebas del concurso. Por otro, la proyección de futuro y su transferencia a la sociedad corre de la mano de los estudios y proyectos que, junto al Instituto de Ciencias de la Construcción Eduardo Torroja (IETcc), la Empresa Municipal de Vivienda y Suelo (EMVS) y el Centro de Innovación de Infraestructuras Inteligentes (CI3), está promoviendo el equipo para reorientar una ciudad obsoleta e ineficiente. Bajo la crisis, esta empresa resulta poco menos que utópica, pero los planteamientos desplegados suponen soluciones que merecen la pena ser exploradas. La revitalización social del barrio, supone la revitalización de sus habitantes, su arraigo y su futuro.

\subsection{Punto de partida}

De los análisis urbanísticos realizados hasta ahora, es conocida la situación de la edificación existente y la necesidad de mejorarla para adaptarla a las exigencias europeas del 2020. Por ello, se plantea la necesidad de creación de una metodología que permita afrontar la regeneración urbana integrada, incluyendo análisis urbanos, sociales, técnicos y económicos, con la aplicación práctica sobre un edificio piloto.

La aplicación de los resultados supone una inmediata transferencia social y tecnológica a la ciudad, además de representar un valor añadido para la potenciación de nuestro sector. En el contexto económico actual, nuestra propuesta resulta prometedora. El concepto no es nuevo; el momento y el lugar sí. La reciente aparición de una nueva legislación en materia de rehabilitación y regeneración urbana, permite entrever cauces por los que pudieran discurrir oportunidades de negocio donde la financiación externa haría viable la rehabilitación.

Entre los objetivos del proyecto se encuentran la unificación de criterios y la recopilación de información sobre otros análisis urbanos realizados, la definición de un método de evaluación de las zonas y condiciones donde una mejora energética y una inversión económica pueda ser rentable, el estudio de una propuesta normativa que permita el desarrollo del proyecto, eliminando barreras y colaborando con las administra- ciones en el desarrollo de propuestas reales de actuación, la evaluación energética de los edificios construidos, la puesta en práctica de nuevos sistemas constructivos que reduzcan el consumo y aumenten el confort de los usuarios, el análisis y cuantificación del coste energético actual y la valoración de la rehabilitación en la reducción de consumos, y sobre todo, el desarrollo de un modelo de gestión de la viabilidad económica.

Es importante precisar que el objetivo de este texto no reside en la presentación de datos y conclusiones sobre la propuesta del proyecto SYMBCITY, ya que se encuentra en periodo de conformación. Responde tan solo a un intento de informar, tanto de las posibilidades de desarrollo como de la expectación que esta idea ha ido suscitando en los diversos foros donde ha sido mostrado, aventurando y sugiriendo métodos, y señalando derroteros por donde pudiera avanzar.

\subsection{La rehabilitación en España}

La rehabilitación urbana ha sufrido varias transformaciones que pueden resumirse en estas fases:

- Recuperación de algunos edificios históricos. La rehabilitación se centraba en mantener algunos hitos culturales importantes de los centros de las ciudades.

- Transformaciones de los centros históricos: Se comenzaron a analizar los centros de las ciudades en su conjunto con la intención de mantenerlos.

- Recuperación de la ciudad existente. La preocupación por la sostenibilidad ambiental y social y la actual situación económica, ha incrementado el interés por la recuperación de los barrios periféricos de nuestras ciudades, que han sido objeto de la mayoría de los programas y ayudas para el fomento de la rehabilitación, teniendo en cuenta criterios de eficiencia energética.

Zaragoza, Madrid o Barcelona (1) albergan ejemplos conceptualmente cercanos a la propuesta SIMBCITY, donde accesibilidad, reducción de la factura energética y cohesión social forman el núcleo de la intervención.

Este tipo de actuaciones se basan en importantes inyecciones de dinero público y por lo general tienen por objeto la mejora de las prestaciones de los elementos de la envolvente térmica, instalaciones de climatización y la inclusión de nuevos núcleos de comunicación vertical para mejorar la accesibilidad. El objetivo de nuestra propuesta es conseguir el máximo de eficiencia y de recursos propios para alcanzarlo, sin desmerecer por supuesto los posibles incentivos y ayudas públicas, pero evitando que éstas se conviertan en el condicionante indispensable para llevar a cabo la rehabilitación.

\section{ANTECEDENTES}

\subsection{Estudios sobre edificios existentes, rehabilitación y eficiencia energética}

Se ha hecho un gran esfuerzo de inversión a través de proyectos de investigación europeos en creación de bases de datos sobre las características de los edificios y las políticas de mejora de su eficiencia energética en los distintos estados de la UE (2) entre ellas podemos destacar:

- BuildingRating (3), que recopila un buen número de ejemplos de escalas de certificación y etiquetados de eficiencia 
energética alrededor del mundo, incluyendo todos los países de la UE.

- EPISCOPE (4) que recoge los resultados de sucesivos proyectos europeos (DATAMINE, TABULA, EPISCOPE) donde se pretende crear bases de datos de tipologías edificatorias definidas como punto de partida para la realización de actividades de monitorización. El objetivo principal es el de realizar un seguimiento del progreso de la eficiencia de la edificación en cuanto al ahorro de energía y los objetivos de reducción del impacto medioambiental con el fin de desencadenar acciones de mejora o correctivas por los actores clave involucrados (políticos, sector industrial, propietarios de edificios, etc.).

- ODYSSEE-MURE (5) que reúne a entidades representantes de los 28 Estados miembros de la UE más Noruega. Su objetivo es vigilar las políticas de eficiencia energética en Europa, utilizando dos bases de datos complementarias: ODYSSEE, sobre indicadores de eficiencia energética y emisiones de $\mathrm{CO}_{2}$, (alrededor de 1.000 series de datos por país) y MURE, sobre las normativas y medidas de fomento de eficiencia energética, incluido su impacto (en torno a 2.000 medidas recogidas).

En lo referente a rehabilitación, proyectos como rethinkingrefurbishment (6), llevado a cabo por el National Refurbishment Centre en Reino Unido, pretenden ofrecer información que permita al sector industrial tomar decisiones y fijar directrices políticas en materia de eficiencia energética, conociendo las medidas más adecuadas para cada tipología constructiva. El portal pretende ser una base de datos en línea que permita buscar casos de estudio. El centro nacional de la restauración también está acumulando en una biblioteca en línea las mejores guías y prácticas en todos los aspectos de la restauración «verde» y de la rehabilitación, así como de la información en acontecimientos y seminarios relacionados con esta materia.

Otras iniciativas se han centrado en la creación de herramientas de ayuda para desarrollar estrategias territoriales de rehabilitación energética, como el modelo SEC (Sustainable Eenergy Cost) propuesto a través del proyecto Factor 4 (7). $\mathrm{El}$ análisis bottom up realizado con la SEC se basa en una tipología de edificio/usuario y en el análisis de edificios representativos: la identificación del óptimo técnico-económico y elaboración de escenarios antes de hacer una extrapolación al conjunto del parque inmobiliario según su tipología (8).

Finalmente, el proyecto que más se aproxima a los objetivos de este estudio es el proyecto SuRE-Fit (9) cuyo objetivo es analizar la mejora de edificios existentes con criterios de eficiencia energética y obtención de recursos financieros mediante la extensión del tejado ${ }^{1}$, realizado durante 2007-2009. Los casos estudiados se limitan a una docena entre Francia, Holanda, Dinamarca, Alemania y República Checa, y los resultados del proyecto indican un alto potencial de aplicabilidad de estas estrategias al resto de la Unión Europea.

Sin ánimo de ser exhaustivos, todo este esfuerzo no parece encontrar su traducción de forma dinámica y eficaz en el sector inmobiliario, por lo menos en España. En estos proyectos la participación española es escasa o nula en muchos de ellos. Si bien en nuestro país se llevan a cabo proyectos a nivel nacional, en el ámbito de la rehabilitación energética de edificios existentes, el objetivo suele estar encaminado a las soluciones técnicas más que a la gestión o la viabilidad económica.

\subsection{Aumento de la edificabilidad. Experiencias recientes}

En nuestro país, salvo en los casos en los que se ha realizado una regeneración urbana completa, donde las edificaciones previas estaban abandonadas y se ha procedido a su demolición o rehabilitación integral, no se han documentado ejemplos en los que se hayan incorporado nuevas superficies construidas a los edificios existentes cuya explotación, permita retornos económicos directos que faciliten la financiación de dicha operación.

Las limitaciones en las normativas municipales y en la mayoría de los casos la colmatación de las edificabilidades en los centros de las ciudades ha impedido que hasta ahora se den con fluidez casos de aumentos de edificabilidad en las rehabilitaciones.

Tan solo se han llevado a cabo actuaciones puntuales en centros de ciudades sobre edificios que tenían alguna protección en sus fachadas y permitían aumentos de edificabilidad, pero con un fin completamente diferente al que planteamos ahora. Estas rehabilitaciones suponían tan solo el mantenimiento de esas partes protegidas y la construcción del resto del edificio casi desde cero, sin tener en cuenta, por supuesto, el aspecto social de los que habitaban el edificio, que normalmente se veían obligados a abandonar sus viviendas. Estas viviendas han sido transformadas, además, en viviendas de lujo, con un fin especulativo.

Con ánimo de encontrar continuidad en el tiempo de los casos estudiados en proyectos como SURE-fit, y de su repercusión en otros sectores (industrial y académico) a continuación se exponen una serie de casos ilustrativos de actuaciones e iniciativas que tienen en cuenta el aprovechamiento del espacio disponible bajo cubierta o la ampliación de una planta en edificios existentes como parte fundamental de la estrategia de intervención

- Rehabilitación de conjunto de edificios residenciales en Viena, Rudolf Zeller Gasse 54; Atelier Heiss Architekten: Esta intervención, completada en 2011, consiste en la rehabilitación de unas 200 viviendas, distribuidas en 12 bloques de 4 plantas, a los que se añade una planta de apartamentos, accesible mediante nuevos núcleos de ascensores adosados a la fachada (Figura 1a)

- Edifico residencial en Kierling, Viena, Architekturbüro Reinberg ZT GmbH: El proyecto, completado en 2012, parte del objetivo de rehabilitar un edifico construido entre 1977 y 1979 y reformarlo acorde al Standard Passivhaus. En la parte superior del edificio existente se añade un nuevo volumen con nuevos apartamentos y se comple-

\footnotetext{
1 Traducción de los autores, en el original: Improvements of existing buildings by better energy performance and new financial resources through roof-top extension».
} 

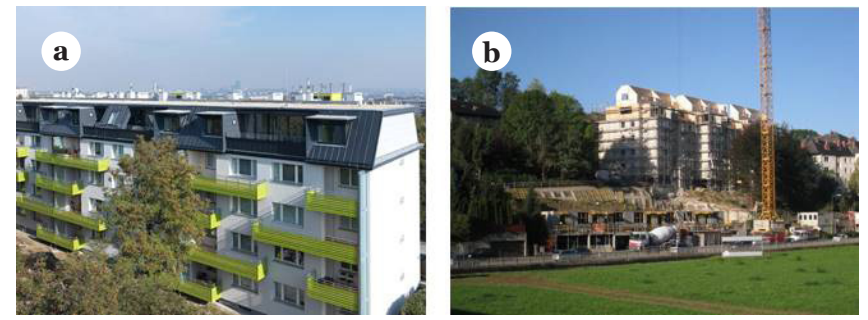

Figura 1. a) Uno de los edificios del conjunto tras la rehabilitación incorporando una nueva planta (10); b) Edificio durante la obra (11).

ta la actuación con la construcción de un nuevo edificio (24 apartamentos después de la renovación). El complejo cuenta con un sistema de ventilación con recuperación de calor, $90 \mathrm{~m}^{2}$ de colectores térmicos y una caldera central de biomasa para conseguir una Aktiv-Solar-Haus (12) (Figura $1 b)$.

No se ha podido documentar si este tipo de iniciativas tienen como base en su planteamiento el incremento de edificabilidad como estrategia para poder acometer el conjunto de la rehabilitación del resto del edificio y en qué contexto legal se han realizado, pero no hay duda de que este tipo de actuaciones, teniendo en cuenta los planes de ordenación Municipal vigentes en la mayoría de nuestro territorio nacional, resultarían prácticamente inviables sin modificaciones sustanciales.

El sector industrial también parece haber tomado buena nota de esta perspectiva. En Francia, la asociación Promotoit ${ }^{2}$ ha planteado esta cuestión, a través de su libro blanco (13), en el que se plantea la estrategia de «sobreelevación» de la cubierta como una de las medidas a tener en cuenta para favorecer la mejora de aislamiento térmico de los edificios. Como ejemplo se menciona un Proyecto de elevación de un edificio en París. Con una superficie edificable de $677 \mathrm{~m}^{2}$. La superficie adicional creada por el proyecto es de $260 \mathrm{~m}^{2}$. La venta de los derechos a construir se valora en unos 2.000.00o de euros, con lo que se va a financiar el trabajo de mejora de la eficiencia energética y actualización del edificio según la normativa vigente, generando una plusvalía de 700.000 euros (14).

En España han surgido algunas iniciativas con esta intención, como la propuesta de la empresa LCT (15) que propone este modelo de negocio para el ensanche de Barcelona. LCT ofrece a los propietarios de los edificios que disponen de edificabilidad remanente, de acuerdo con las normativas vigentes, un diálogo cuyo objetivo es alcanzar un acuerdo de beneficio mutuo: la propiedad puede asumir el mantenimiento y la actualización de la finca gracias a la inversión de LCT, mientras que ésta obtiene un emplazamiento para el desarrollo de viviendas en ático. Así, tanto quienes prefieran alquilar como vender los derechos de edificabilidad remanente de sus azoteas contarán con una respuesta a sus demandas. Los primeros ejemplos ya se pueden ver en la ciudad a través de su página web (15).
Otro ejemplo es el planteado por Modular Projects, que ha realizado su primera actuación en Alicante basada en la construcción industrializada modular con estructura de madera (16).

En ambos casos se trata de actuaciones puntuales, en un único edificio, en los que además el incremento de superficie construible es posible gracias a que el edificio existente no había consumido la edificabilidad asignada por el planeamiento. Tampoco parece que el planteamiento original de estas propuestas surja de la necesidad del propio inmueble existente.

En el ámbito académico, los concursos de arquitectura para estudiantes han servido igualmente para plantear este tipo de estrategias en distintos ámbitos. En el Concurso de Universidades «Ciudad sostenible: la rehabilitación como herramienta» promovido y propuesto por GBCe y desarrollado por ASA (Asociación Sostenibilidad y Arquitectura), en concordancia con el Congreso SB1omad, el proyecto ganador (ex aequo) «Bellvitge $64 \% »^{3}$, plantea la sustitución de viviendas en planta baja y su reubicación en la cubierta, como estrategia de rehabilitación sostenible en un conjunto residencial de esta localidad, como ejemplo representativo de los polígonos de vivienda de la Región Metropolitana de Barcelona (17).

En el ámbito de la presente edición del concurso SDE2014, motivo de este trabajo, es significativo que otros tres equipos europeos de Alemania y Dinamarca -Equipo ONTOP, de la universidad de Applied Sciences Frankfurt (18), Equipo ROOFTOP, formado por Technische Universität Berlin (TU Berlin) y the University of the Arts Berlin (UdK Berlin) (19), y equipo DTU, de la Technical University of Denmark en Copenague (20)-, han elegido la cubierta de los edificios existentes como ubicación de su propuesta de vivienda. En todos los casos se plantea como objetivo la redensificación de la ciudad existente y dotar de un suministro adicional de energía renovable al edificio huésped. En estos casos no se incide expresamente en el objetivo de mejorar la envolvente térmica del edificio existente, quizás porque ésto es algo ya asumido y realizado en muchos de los conjuntos residenciales en estos países, ni en la componente financiera, ya que en algunos casos estos espacios bajo cubierta existen en las tipologías residenciales colectivas y lo que se plantea es su sustitución o adecuación.

A la vista de lo expuesto, quedan por tanto dos cuestiones pendientes de resolver: ¿Cómo acometer este tipo de actuaciones cuando el planeamiento actual no lo permite? y ¿cómo conseguir que esto sea aplicable a conjuntos residenciales de gran escala?

\subsection{Edificabilidad vs Eficiencia energética}

En este sentido parece que la solución debe pasar por un reajuste de la normativa urbanística que permita, aunque sea

\footnotetext{
${ }^{2}$ La asociación Promotoit se crea en 2005 y agrupa a nueve empresas del sector industrial especializadas en la producción de materiales de construcción para las cubiertas de los edificios (ETERNIT, IMERYSTOITURE, ISOVER, SIPLAST, TERREAL, UNILIN, VELUX, VM-ZINC, WIENERBERGER).

3 Profesores: Iván Muñiz; Profesor titular del departamento de Economía Aplicada de la UAB, Dani Calatayud; profesor asociado del departamento de Urbanismo y Ordenación Territorial de la UPC y Coque Claret profesor asociado del departamento de Proyectos de Arquitectura de la UPC.
} 
temporalmente, un aumento de la edificabilidad en determinadas zonas a cambio de la justificación de la mejora de la eficiencia energética. Esta solución, se recoge parcialmente en Ley 8/2013, de 26 de junio, de rehabilitación, regeneración y renovación urbanas que en su artículo 10 menciona la posibilidad de ocupar las superficies de espacios libres o de dominio público así como las superficies comunes de uso privativo, tales como vestíbulos, descansillos, sobrecubiertas, voladizos y soportales, tanto si se ubican en el suelo, como en el subsuelo o en el vuelo, cuando no resulte viable, técnica o económicamente, ninguna otra solución para garantizar la accesibilidad universal o la realización de obras que consigan reducir al menos, en un 30 por ciento la demanda energética anual de calefacción o refrigeración del edificio en el caso de utilizar determinadas soluciones constructivas para mejorar la envolvente térmica ${ }^{4}$. De cara a nuestra propuesta parece insuficiente a los efectos de conseguir los recursos financieros necesarios.

En este sentido, las propuestas realizadas en algunas regiones del Norte de Italia, como el Trentino alto-Adige, parecen estar un paso por delante en la materialización de esta posibilidad. Así, la Deliberacione de aplicación de la Directiva de Eficiencia Energética en Edificios 2010/31/UE del Parlamento Europeo ${ }^{5}$ recoge, en su artículo 11, las siguientes propuestas:

\section{Sistema de incentivos Bonus cubatura:}

- Como medida para favorecer la eficiencia energética, el volumen edificado permitido para el edificio rehabilitado se incrementará temporalmente hasta final de 2014, un $10 \%$ para clase Casa Clima B y un 15\% para clase Casa Clima A, y un $10 \%$ en este último caso hasta 2019.

- En caso de rehabilitación de un edificio residencial de dimensiones importantes, el volumen edificado se puede aumentar hasta $200 \mathrm{~m}^{3}$, si el edificio presenta un volumen edificado sobre rasante de al menos $300 \mathrm{~m}^{3} \mathrm{y}$ si mediante las obras se consigue aumentar la calificación energética Casa Clima al menos a Casa Clima C. Para tal fin se admite un aumento de la altura máxima permitida de hasta 1 metro.

- La edificabilidad admisible para uso vivienda, vivienda protegida y actividad terciara de los edificios existentes, en zona residencial y en zona industrial, puede aumentarse un $20 \%$ y se admite un aumento de 3 metros de la altura máxima permitida.

\section{METODOLOGÍA}

Después del recorrido trazado en el capítulo anterior, se ha podido observar la existencia de una importante cantidad de iniciativas, europeas y españolas, que expresan la necesidad de acometer este tipo de intervenciones desde una óptica holística. Surge entonces la necesidad de establecer un método, la necesidad de ordenar pautas, objetivos e instrumentos, que aseguren la eficacia de los planteamientos.

\subsection{Objetivos y planteamientos}

\subsubsection{Marco legal}

Un nuevo marco legal en el campo de la rehabilitación queda definido por la Directiva 2012/27/UE del parlamento europeo y del consejo, de 25 de octubre de 2012, relativa a la eficiencia energética que impulsa un ahorro energético de un $20 \%$ para 2020 y la Ley de rehabilitación, regeneración y renovación urbanas de 8/2013, de 26 de junio, que establece mecanismos que posibilitarían la financiación externa para una intervención rentable. Son luces en el túnel que permiten albergar esperanzas. Tanto el Real Decreto 233/201, por el que se regula el Plan Estatal de fomento del alquiler de viviendas, la rehabilitación edificatoria, y la regeneración y renovación urbana 2013-2016, como el Real Decreto 235/2013, por el que se aprueba el procedimiento básico para la certificación energética de los edificios abundan en esta dirección. Resta mucho camino legislativo aún.

Hasta ahora la rehabilitación era solo posible con aportaciones de los propietarios y subvenciones de las Administraciones Públicas. El recurso en el que basamos la viabilidad de nuestra propuesta es el aumento de edificabilidad para financiar la rehabilitación del edificio existente. El marco normativo actual tiende a facilitar operaciones que eliminan obstáculos en la puesta en práctica de estos objetivos y propician además la generación de ingresos propios para hacer frente a las mismas. La ley 8/2013 hace referencia a este aspecto, respecto a dos tipos de actuaciones preferentes: la mejora de la accesibilidad y la eficiencia energética. Para poder llevar a cabo la financiación de estas actuaciones, se incorporará un informe o memoria de sostenibilidad económica, que contendrá, entre otras cosas, las modificaciones sobre el incremento de edificabilidad o densidad y la posible introducción de nuevos usos.

Es decir, el camino está trazado, pero desde nuestra perspectiva, se trata aún de una realidad incipiente, con escaso calado y ambición contenida. Insuficiente. Esto, justificaría de por sí la investigación y la metodología propuesta.

Dentro de los objetivos del proyecto, se encuentra la necesidad de obtener información fidedigna sobre el alcance que tendría la aplicación de los supuestos de aumento de edificabilidad y la relación de parámetros que harían posible (y sobre todo rentable) la actuación. Supongamos, por poner un ejemplo, que los datos económicos del modelo de edificación elegido como «colono» o «huésped» (en función de las necesidades vecinales, la tipología del edificio, la calidad del barrio, las estrategias desplegadas,...), contemplaran su viabilidad (económica y urbana), para un incremento de edificabilidad por encima de lo inicialmente estimado por la reglamentación; es decir, supongamos que la operación resultara viable y asequible para la incorporación de dos niveles adicionales sobre la azotea, en lugar de tan solo uno, y este incremento rebasara las expectativas iniciales de la reglamentación. Estas cuestiones pueden y deben ser estudiadas, visualizadas y debatidas, y el proyecto SYMBCITY y su desarrollo metodológico pretende ponerlo de manifiesto.

\footnotetext{
${ }^{4}$ Artículo 10. Reglas básicas para la ordenación y ejecución de las actuaciones.

${ }_{5}^{5}$ BOLLETTINO UFFICIALE - AMTSBLATT DELLA REGIONE AUTONOMA DER AUTONOMEN REGION TRENTINO-ALTO ADIGE/SÜD-

TIROL 12 marzo 2013. Supplemento n. 2 (traducción de los autores).
} 


\subsubsection{Viabilidad urbana y constructiva}

No existen grandes inconvenientes para proyectar y ejecutar las técnicas de intervención necesarias en este tipo de actuaciones; sobre todo, no existen (entiéndase el sentido de la exageración) propuestas que no puedan ejecutarse con la incorporación de los recursos económicos adecuados. Éste, representa uno de los temas esenciales de estudio y desarrollo de la actuación. Pero existen preguntas previas: ¿qué tipo de producto necesito?, ¿cuál es el perfil del usuario que demandará mi producto?, etc. De otro modo: ċlas azoteas acogerían viviendas, escuelas, mercados, garajes, centrales de distrito de producción energía...?

Resulta evidente que las respuestas vienen de la mano de un estudio previo, que identifique las carencias del tejido urbano; tras el análisis de su morfología, movilidad, su complejidad y cohesión social, su metabolismo urbano..., y cuantos indicadores arrojen luz sobre sus perspectivas de futuro, se podrán proponer actuaciones que apuntalen un modelo urbano de desarrollo sostenible. Resultaría relevante el estudio de la vulnerabilidad (21) entendida como «aquel proceso de malestar producido por la combinación de múltiples dimensiones de desventaja, en el que toda esperanza de movilidad social ascendente,...» «...es contemplada como extremadamente difícil de alcanzar». Probablemente, los ejemplos escogidos, no respondan a situaciones extremas de marginación, pero arrojarían luz sobre situaciones difíciles en el actual contexto social y económico. Con todo esto, como se puede prever, la comunicación previa con asociaciones y representantes sociales, como parte activa del proceso, resulta esencial.

En el corto periodo de tiempo transcurrido desde el inicio de este ambicioso proyecto, se han ido poniendo las bases sobre las que poder cimentar procedimientos posteriores, identificando, por ejemplo, los objetos de atención prioritaria: el tejido residencial urbano de las periferias de las grandes ciudades acoge durante el desarrollismo de los años 50-60 una vasta cantidad de inmigrantes rurales atraídos por las perspectivas de futuro que deparan tanto el sector industria como el sector servicios, y que demandan abundante mano de obra. Son acogidos en edificaciones que examinadas bajo parámetros actuales de confort y habitabilidad resultan claramente insuficientes.

La colonización de las azoteas no resulta una propuesta novedosa (ver apartado 2), y su viabilidad técnica dependerá de las condiciones de partida de cada objeto individual. La capacidad portante de las estructuras existentes y su grado de deterioro originarán actuaciones que permitirán el apoyo de los nuevos habitáculos o la necesidad de buscar soportes más firmes en terrenos circundantes.

En cualquier caso, la actividad «colonizadora» permitirá implantar toda la tecnología de climatización, abastecimiento y generación de energía de los espacios «huéspedes», que en el ánimo de deslizarse por la fachada, desde la cubierta al suelo, revestirá de una segunda piel al edificio existente; obtendrá con ello, una considerable mejora de sus rendimientos energéticos y una nueva concepción estética acorde con los tiempos actuales. Balcones, miradores, invernaderos, terrazas... podrán entonces sumarse como estrategias pasivas de acondicionamiento ambiental pasivo, al balance energético de la solución adoptada; todo ello, sin merma de las soluciones convencionales, que los sistemas de mejora en la resistencia térmica de los paramentos, pudieran aportar.

Se consigue con todo ello una completa y compleja revitalización de la edificación en el más amplio sentido de la palabra, y sobre todo la adquisición de un paquete de soluciones, cercano a la habitabilidad que una obra nueva pudiera deparar.

\subsubsection{Viabilidad económica}

Llevando el discurso a términos de mercado (y tomándolo en sentido social y no con un ánimo única y exclusivamente mercantilista), supongamos que, después de llevar a cabo un exhaustivo análisis urbano, hemos logrado identificar el producto necesario y el usuario al que va destinado; sabemos cómo fabricar el producto, cuánto cuesta y por cuanto podemos ponerlo en mercado (después de sopesar las diversas téc-

\section{SymbCity}

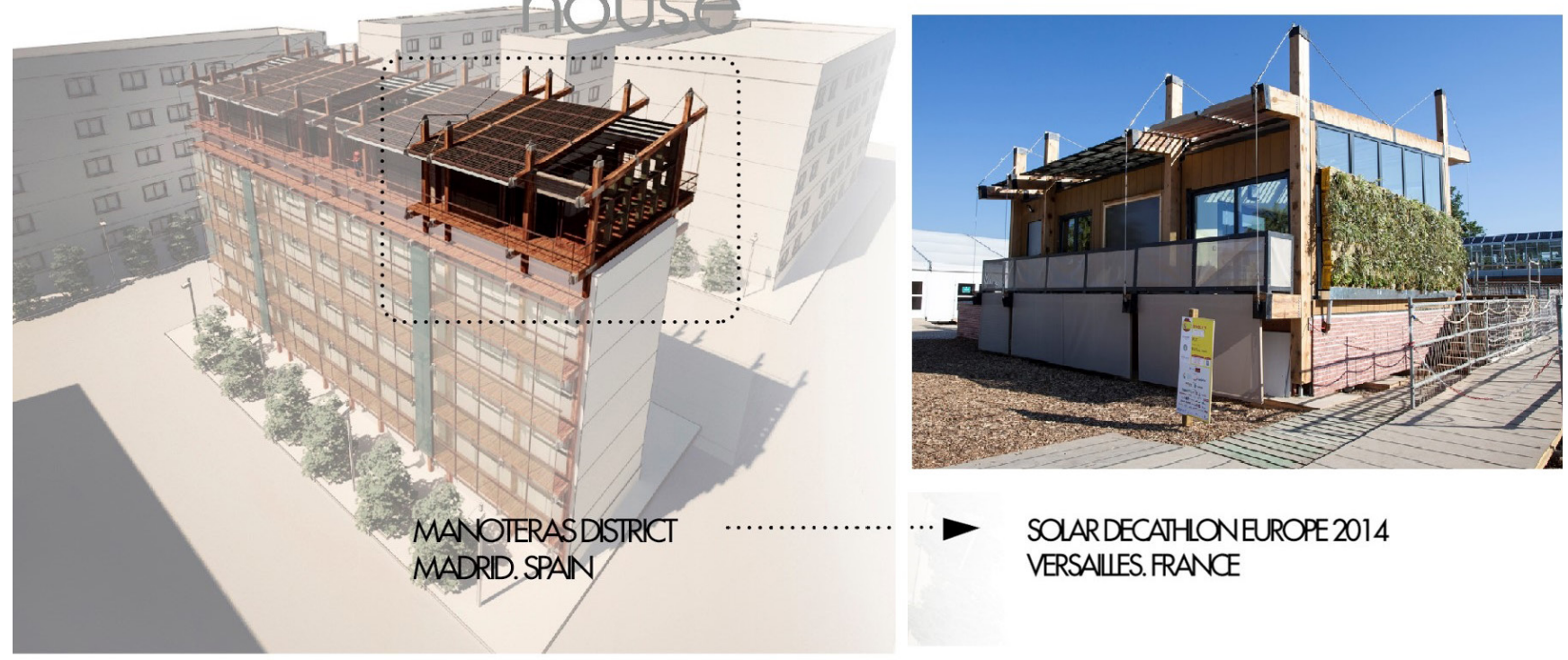

Figura 2. Equipo Plateau Team, proyecto Symbcity, SDE2014: repercusión entre simbiontes, aportaciones mutuas. 
nicas constructivas aplicables); finalmente debemos procurar que resulte económicamente viable. El estudio económico producirá bucles con el Área Técnica de Estudios hasta que la solución constructiva adoptada y los recursos económicos exigibles originen el producto adecuado. Desde los supuestos planteados se podrá operar tanto desde la iniciativa privada como desde la promoción pública. Las administraciones llegarían a acuerdos con las comunidades de propietarios que permitirían la rehabilitación del edificio a cambio de la cesión de la azotea para la realización de operaciones urbanas consensuadas. Este discurso pertenece a trabajos previstos en proyecto, que se consolidarán cuando se habiliten los recursos económicos imprescindibles para su progreso.

Es preciso resaltar que la razón y preocupación vital de este proyecto, «aquí y ahora», momento y lugar, consiste en incorporar una serie de instrumentos y procedimientos en el discurso urbano, que logren paliar el deterioro de sus edificaciones (sobre todo en el ámbito del aprovechamiento de los recursos energéticos), y consigan renovarlas conforme a los actuales criterios de confort y habitabilidad; todo ello en un contexto de crisis, que repercute sobre todo en una población que no posee recursos económicos suficientes para subsanar estas deficiencias.

Es ahora cuando existen novedades de consideración con respecto a los antecedentes detectados. Es ahora cuando la carencia de recursos económicos de la población, las necesidades de regeneración y revitalización de construcciones obsoletas, las sugerencias de una incipiente normativa, y el trabajo conjunto, simultáneo y coordinado, de las áreas urbana, técnica y económica, como espejos de las esferas medioambiental, social y económica de la sostenibilidad, anuncian un método y unos resultados que pueden colaborar a la regeneración de barrios deprimidos con escasas perspectivas de futuro. Representa, además, en el estado actual, un modelo de transferencia de conocimiento, de gran calado social, entre la Universidad y la Ciudad.

\subsection{Plan de trabajo. Organigrama}

Cualquier método debe propiciar una sincronización de tareas en base a un objetivo preestablecido. Las tareas son ejecutadas por las Áreas de Viabilidad y los objetivos están gobernados por Estrategias de Actuación.

Definir las Estrategias de Actuación supone por tanto abordar aquellas materias, precisar aquellas pautas, que deben conducir al cumplimiento de los objetivos planteados. Y esto debe hacerse desde el Comité de Coordinación del proyecto. Aquí estará representada cada una de las áreas de investigación y desarrollo, nombradas como Áreas de Viabilidad. Las estrategias tienen como referente los pilares de la sostenibilidad en sus tres ámbitos: medioambiental, social y económico.

\section{Estrategias medioambientales:}

- Reducción de la demanda energética a través de la intervención en la envolvente térmica. Implantación de sistemas pasivos de acondicionamiento ambiental.

- Implantación de instalaciones de climatización, ventilación e iluminación eficaces y eficientes.

- Incorporación de instalaciones productoras de electricidad.
- Utilización de materiales de bajo impacto ambiental.

- Autosuficiencia hídrica de la demanda urbana. Gestión eficaz del agua.

- Gestión adecuada de los Residuos Sólidos Urbanos. Puntos limpios.

Estrategias sociales:

- Revitalización del espacio urbano. Mejora de la calidad de vida de sus habitantes. Arraigo y proyección de futuro.

- Estrategias de movilidad. Dotación de recursos e instrumentos para la accesibilidad, de cualquier sector de población. Creación y distribución geográfica de servicios y equipamientos.

- Participación y cohesión social. Concienciación de la población en hábitos de reducción de impactos.

- Utilización de recursos locales. Fomento del empleo e intercambio de productos y servicios en un entorno próximo. Estrategias de ocio y activación de sectores no ocupados laboralmente.

- Estudio de la normativa existente y propuestas de modificación legislativa que permita actuar con flexibilidad sobre la redensificación sostenible de las ciudades.

Estrategias de financiación:

- Definición del perfil del usuario del producto en venta.

- Definición del modelo de negocio que permite poner el producto en el mercado. Identificar un comprador y un modelo de consumo.

- Aportaciones de subvenciones públicas.

- Amortizaciones en las facturas de los recursos optimizados (energía, agua...).

- Revalorizaciones por el aumento de la calidad de la edificación.

- Revalorización del $\mathrm{m}^{2}$ construido por la revitalización del espacio urbano.

Para analizar la situación actual, se ha considerado de interés desglosar áreas de trabajo especializadas. No pueden ni deben ser parcelas independientes. Constituyen áreas de investigación y desarrollo, que atienden a los objetivos trazados desde el Comité de Coordinación, y que están en contacto permanente. La consolidación de las áreas de Viabilidad Urbana y Constructiva, de Viabilidad Económica, Gestión de la Intervención y Administración de Recursos, y de Herramientas de Análisis y Diagnóstico y Comunicación, asegurará la eficacia del método.

\subsection{Funcionamiento de la estructura organizativa}

Una vez reconocida una cierta estructura jerárquica de funcionamiento, debe procurarse la conexión horizontal y transversal de las áreas de trabajo, en aras de una eficaz colaboración. Decidido el objeto de trabajo (un determinado entorno urbano) y consensuadas como «compromiso fundacional» las Estrategias de Actuación, el Área Viabilidad Urbana en colaboración con el Área de Gestión de la Intervención y Administración de Recursos, procederá a establecer un calendario de actuaciones que comenzarán por la demanda de participación ciudadana a través de los organismos de representación que sus habitantes se hayan otorgado, como por ejemplo, las asociaciones vecinales. No pueden faltar, en estos compromisos iniciales, la participación de la administración local, encargada de canalizar las inquietudes y gestionar recursos que promuevan soluciones. 
Se estudiarán necesidades y carencias, decidiendo qué tipo de productos o servicios se incorporan a las azoteas de los edificios existentes, bien sean del tejido residencial (estudios, apartamentos,...), del sector servicios, (oficinas, aparcamientos, bibliotecas, ambulatorios o guarderías,...) o tal vez, empresas de servicios energéticos con capacidad de gestionar y promover el uso de energías renovables para la colectividad. También podrían moverse piezas del entramado original (las plantas bajas podrían liberar suelo, para otros usos) o desaparecer construcciones de alto impacto y baja densidad, para la dotación de espacios comunes de relación social. Estamos hablando del producto o servicio de mercado, y desde luego, del perfil del usuario.

Recogerá el testigo el Área de Viabilidad Técnica, que propondrá soluciones que serán valoradas, evaluadas y calificadas por el Área de Viabilidad Económica, quien a su vez devolverá esta información al Área Técnica y Urbana con propuestas de mejora de rendimientos y amortización de la inversión. Si por ejemplo, el análisis económico detecta la imposibilidad (o riesgos) de puesta en mercado del producto, pero augura buenos resultados con la incorporación de un segundo nivel (por ejemplo), porque detecta una demanda aceptable, las Áreas Urbana y Técnica deben resolver sobre la viabilidad de la nueva propuesta y advertir de los peligros que estas incorporaciones pudieran deparar. Después de recorrer los ciclos de ajuste precisos, debe surgir la propuesta definitiva.

\subsection{El papel de la comunicación. SDE2014}

La difusión y comunicación constituye un elemento esencial impulsor del proyecto, en tanto dé lugar a sugerencias, iniciativas y propuestas de actuación. Como se verá posteriormente la elección del modelo de implantación tuvo como desencadenante principal esta consideración.

La divulgación de resultados, aciertos y fracasos, tiene también presencia relevante en el organigrama inicial. Actúan como catalizadores de futuras actuaciones y colaboran en su posible extensión a otros ámbitos.

Desde esta óptica, el gran impulsor del proyecto es el Concurso Internacional Solar Decathlon 2014, como ya se ha puesto de manifiesto en la presentación inicial haciendo referencia a los dos caminos complementarios, simbióticos. La cantidad de foros en los que se ha presentado, los debates surgidos, las sugerencias y el entusiasmo con el que ha sido recogido por estamentos y sectores variopintos, arroja alguna luz sobre sus posibilidades de desarrollo. La relevancia de este carácter didáctico ya ha sido puesta de manifiesto en foros de impacto científico como demuestran varios de los artículos publicados en el número monográfico de Energy and Buildings de próxima aparición (22) (23).

La propuesta de actuación concreta, en consonancia con los planteamientos expresados en líneas anteriores, consiste en la creación de un nivel de viviendas adicional sobre la cubierta de un edificio existente, al que se adapta mediante una estructura exterior, y cuyos detalles se pueden consultar en la página web del proyecto (24).

\subsection{Implantación y generalización del método}

Como ejemplo dinamizador del método, que supone tanto su puesta en acción como su procedimiento de revisión y adecuación, se ha tomado Manoteras, barrio periférico de la zona norte de Madrid, que reúne todas las características que hacen de la propuesta ejemplo y modelo. Supone un encaje perfecto en su concepción y objetivos. Se trata, en sus orígenes, de un poblado dirigido de los años 6o, que acoge a una población rural que busca acomodo en las grandes ciudades y no dispone de grandes recursos económicos.

Aunque concurren tipologías diversas en función de su año de construcción, nos centramos en el poblado dirigido de 1958, con grupos residenciales «que combinan unifamiliares adosadas y bloques de cinco alturas enlazados por testeros en una trama ortogonal abierta que permite crear calles peatonales y pequeñas plazas entre ellos, siguiendo la distribución libre habitual en otras promociones oficiales del momento» (25).

Sus condiciones de habitabilidad, con una envolvente sin aislantes, con un tratamiento de huecos individualizado en la mejor de las ocasiones, permite asegurar una intervención exitosa tanto en las condiciones de confort como en su factura energética.

Otras comunidades presentan condiciones de trabajo similares, pero su elección, asesorados por la EMVS (Empresa Municipal de Vivienda y Suelo) de Madrid, socio del programa, corresponde a un motivo fundamental que caracteriza al barrio: el dinamismo de su asociación de vecinos. Como ya se ha señalado, la participación, comunicación y difusión de las acciones del método constituyen su columna vertebral.

Hasta el momento, se han desarrollado estudios incipientes que auguran excelentes perspectivas de futuro. Junto con el IETcc (Instituto de Ciencias de la Construcción Eduardo Torroja) se están estudiando, mediante monitorización, algunas de las viviendas de los bloques modelos. Desde el Área de Herramientas de Análisis y Diagnóstico, se están recogiendo y procesando estos datos, que nutrirán las futuras actuaciones.

Paralelamente con la EMVS, dentro del área de Viabilidad Económica, se están desarrollando procedimientos de estudios financieros sobre estándares de intervención, que permitan extender los resultados del método a otras comunidades similares.

Además, fruto de la actividad académica desplegada en torno a la propuesta, se han realizado en ambas universidades trabajos fin de grado que recogen análisis relevantes. En uno de ellos (26), tras un análisis previo del parque de viviendas de Castilla La Mancha (CLM), se ofrece información sobre los edificios de las seis ciudades más importantes de CLM que podrían recibir el prototipo diseñado por Plateau Team, y su viabilidad económica en comparación con una intervención estándar.

Alumnos de la Universidad de Alcalá (UAH), pertenecientes al Área de Herramientas de Análisis y Diagnóstico, han trabajado sobre el barrio de Manoteras en Madrid, en sentidos diversos. Por un lado, recabando información sobre un edificio tipo, con estudios climáticos, morfológicos, constructivos y termográficos, que con la utilización de aplicaciones tales como Lider, Calener y Design Builder, obtienen datos sobre los beneficios de una futura intervención en la envolvente térmica y en la mejora de instalaciones de calefacción y ACS. Terminan por realizar estudios de viabilidad económica y amortización de la inversión (27), computando incluso ayudas estatales (programa PAREER). Sus conclusiones se han transmitido en reunión convocada por la Asociación de vecinos y difundida por órganos de comunicación propios (Radio Enlace). 
Estos breves apuntes son tan solo la punta del iceberg. Creemos en su largo recorrido y en las vías que abre.

\section{ESTIMACIONES ECONÓMICAS PREVIAS}

Para mejorar el parque existente es imprescindible realizar un análisis de la viabilidad económica de cada intervención. Contamos con el inconveniente de que los barrios que requieren un mayor grado de rehabilitación están habitados generalmente por poblaciones de menos recursos.

Hasta ahora la rehabilitación era solo posible con aportaciones de los propietarios y subvenciones de las Administraciones Públicas, que en ocasiones alcanzaban hasta el $75 \%$ del coste de las actuaciones. El nuevo modelo exige que las nuevas operaciones generen sus propios recursos. El Plan Estatal contempla unas subvenciones máximas, que no podrán superar el 35\% del coste subvencionable, ni exceder de 11.000 euros por vivienda. La cuantía máxima se calculará multiplicando por el número de viviendas y por cada $100 \mathrm{~m}^{2}$ de superficie útil de locales del edificio, que consten en la escritura de división horizontal o, en su defecto, en el registro de la propiedad o en el catastro, las ayudas unitarias establecidas a continuación:

- Hasta 4.00o euros para las actuaciones de conservación,

- 2.00o euros para las actuaciones de mejora de la eficiencia (5.00o euros si se reduce en un 50\% la demanda energética del edificio),

- 4.00o euros para las actuaciones de mejora de la accesibilidad.

Con la nueva Ley se establecen mecanismos que permitirán obtener financiación externa para que la rehabilitación sea rentable. Una Memoria de Viabilidad Económica acompañará a cada actuación y recogerá reglas excepcionales para vincular incrementos de edificabilidad o densidad, así como cambios a las distintas operaciones de rehabilitación, regeneración y/o renovación urbanas, siempre que se mejore la eficiencia energética y accesibilidad de los edificios.

Nuestra propuesta pretende cubrir ese $50 \%$ restante del coste de una rehabilitación que reduzca al menos hasta un $25 \%$ del coste total la aportación de los propietarios.

Pero, ¿̇uándo es posible alcanzar estos números que hagan una intervención rentable económicamente?

Para contestar a estas preguntas hemos analizado una serie de intervenciones en edificaciones reales de diferentes localizaciones de Madrid, estimando unos números iniciales medios de rehabilitación. Sobre ellas se plantean una serie de mejoras y ampliaciones:

- Aumento de edificabilidad en cubierta, superficie que sale a la venta.

- Aumento de edificabilidad en cada planta, mejorando el confort y la eficiencia energética de las edificaciones existentes.

- Mejora mínima en las instalaciones con la incorporación de energía solar térmica.

- Mejora en la accesibilidad con la incorporación de ascensores donde son necesarios.
Mediante la confección de fichas de trabajo, se analizaron estimaciones de cálculo referidas a entornos urbanos dispares:

- Manoteras (5 alturas, 10 vecinos y un 20\% de superficie ampliable),

- Lavapiés (3 alturas, 16 vecinos y un $33 \%$ de superficie ampliable),

- Castellana (10 alturas, 73 vecinos y un 10\% de superficie ampliable),

- Madrid Río (12 alturas, 44 vecinos y un 8\% de superficie ampliable).

De estos 4 primeros casos reales se observó cómo el valor de mercado es el parámetro más determinante. Además un valor inferior al 10\% de superficie ampliable hace que la intervención no sea rentable, ya que necesitaría de la aportación de más del $50 \%$ por parte de los propietarios.

A estos casos de estudio reales les modificamos el valor del precio de venta hasta mantenerlo constante para poder comparar la tipología y valorar el resto de parámetros.

Ante un mismo precio de venta de la superficie ampliable llegamos a la conclusión de que la superficie mínima ampliable para que la operación sea viable económicamente es del $20 \%$ de la superficie total. Además un mayor número de plantas también perjudica a la viabilidad de la intervención (Figura 3).

\section{CONCLUSIÓN}

La metodología propuesta aporta una visión integral a nivel de barrio de esta estrategia de redensificación que hasta
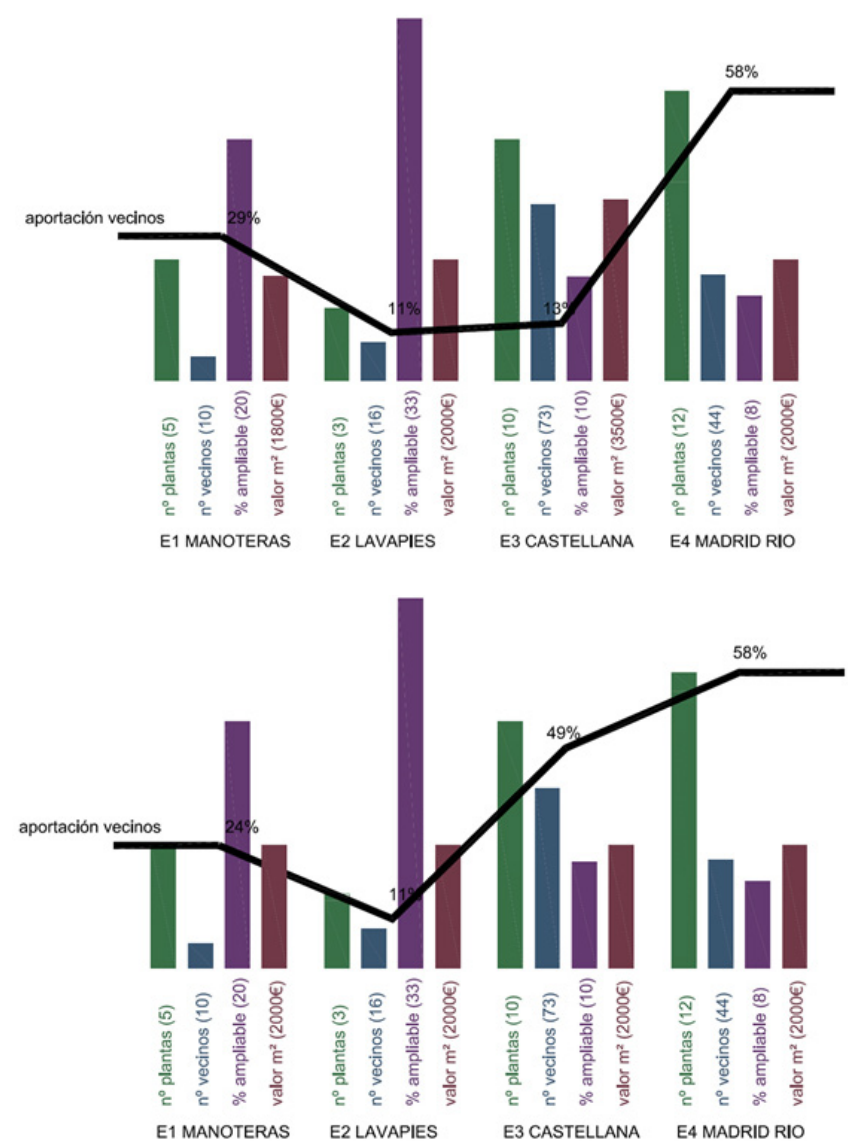

Figura 3. Valores comparativos de aumento de edificabilidad eficaz. 
ahora solo se ha aplicado a rehabilitaciones de edificios sin ampliación de superficie construida de forma relevante. Los primeros análisis demuestran que este tipo de intervención puede ser viable en conjuntos de viviendas homogéneos a gran escala, que permitan la réplica de los prototipos de viviendas y soluciones constructivas de forma sistemática.

\section{REFERENCIAS}

(1) Rubio del Val, J. (2011). Rehabilitación Urbana en España (1989-2010). Barreras actuales y sugerencias para su eliminación. Informes de la Construcción, 63(Extra): 5-20, doi: http://dx.doi.org/10.3989/ic.11.06o.

(2) Data Hub for the energy performance of buildings. http://www.buildingsdata.eu/data-sources.

(3) Building rating disclosure policies and programs. http://www.buildingrating.org.

(4) European Project EPISCOPE. http://episcope.eu.

(5) ODYSSEE-MURE Project. http://www.odyssee-mure.eu/.

(6) National Refurbishment Centre. http://www.rethinkingrefurbishment.com/.

(7) Sustainable Urban Development European Network. http://www.suden.org/en/european-projects/factor-4/.

(8) Charlot-Valdieu, C., Outrequin, P. (2011). An approach and a tool for setting sustainable energy retrofitting strategies referring to the 2010 EPBD. Informes de la Construction, 63(extra): 51-6o, doi: http://dx.doi.org/10.3989/ic.11.064.

(9) SURE-Fit Project. http://www.sure-fit.eu/.

(10) Atelier Heiss Architekten. http://www.atelier-heiss.at/projekto1/index.php?lang=2\&idcatside=188.

(11) Architekturbüro Reinberg. http://www.reinberg.net/architektur/132/fotos.

(12) Reinberg, G.W. (2011, septiembre). «Solar-Aktiv» in der «Sanierung» und «Die aktive Solarstadt» «Aktiv-Solarhaus». En 3rd Symposium Ostbayerisches Technologie-Transfer-Institut (pp. 32-39,148-155). Regensburg: Ostbayerisches Technologie-Transfer-Institut.

(13) El tejado, soluciones para liberar la producción de viviendas. http://www.promotoit.org.

(14) Le Moniteur. (4 de noviembre de 2012). Sur ou sous les toits, des solutions pour produire plus de logements mieux. Le Moniteur. http://www.lemoniteur.fr/145-logement/article/actualite/19413328-sur-ou-sous-les-toits-des-solutionspour-produire-plus-de-logements-mieux-isoles.

(15) La casa por el tejado. http://www.lacasaporeltejado.eu.

(16) Gallardo, A.G. (16 de noviembre de 2012). Una casa en el tejado. El Mundo. http://www.elmundo.es/elmundo/2012/11/15/suvivienda/1353008415.html.

(17) González Díaz, M.J. (2011). Concurso de Universidades - SB1omad «Ciudad sostenible: la rehabilitación como herramienta». Informes de la Construcción, 63(EXTRA): 125-141, doi: http://dx.doi.org/10.3989/ic.11.059.

(18) Equipo ONTOP, de la universidad de Applied Sciences Frankfurt am Main. http://www.ontop2014.de.

(19) Project ROOFTOP. Technische Universität Berlin (TU Berlin), University of the Arts Berlin (UdK Berlin). http://www. teamrooftop.de.

(20) Solar Decathlon Europe 2014. http://solardecathlon.dk/wordpress.

(21) Hernández Aja, A. (2007). Métodos del Análisis Sociodemográfico. Cuadernos de Investigación Urbanística, 2007(53): 8-15.

(22) Ripper Kós, J., Mayer de Souza, B. (2014). Educating home users through a solar house: the Ekó House experience. Energy and Buildings, 83:181-185, doi: http://dx.doi.org/10.1016/j.enbuild.2014.03.080.

(23) Navarro, I., Gutiérrez, A., Montero, C., Rodríguez-Ubiñas, E., Matallanas, E., Castillo-Cagigal, M., Porteros M., Solórzano, J., Caamaño-Martín, E., Egido, M.A., Páez, J.M., Vega, S. (2014). Experiences and methodology in a multidisciplinary energy and architecture competition: Solar Decathlon Europe 2012. Energy and Buildings, 83:1-7, doi: http:// dx.doi.org/10.1016/j.enbuild.2014.03.073.

(24) Plateau Team of Solar Decathlon Europe 2014. http://www.plateauteam.com.

(25) Estudio previo para la declaración del ARI de Manoteras. Empresa Municipal de Vivienda y Suelo. Cap II. Memoria Histórica.

(26) Cuevas, E., López, L. (2013). Proyecto de investigación sobre los edificios existentes de Castilla La Mancha y la comparación de dos tipos de intervención en el edificio (Proyecto Fin de Grado). Cuenca: Escuela Politécnica de Cuenca.

(27) López, J., Fernández, L., Carpintero, S. (2014). Rehabilitación energética en un bloque de viviendas en Manoteras. (Trabajo Fin de Grado). Alcalá de Henares: Escuela de Arquitectura Técnica de la Universidad de Alcalá. 